\title{
Kinetic Monte Carlo Investigation of the Effects of Vacancy Pairing on Oxygen Diffusivity in Yttria-Stabilized Zirconia
}

Kinetic Monte Carlo Investigation of the Effects of Vacancy Pairing on Oxygen Diffusivity in Yttria-Stabilized Zirconia

Brian S. Good

Materials and Structures Division

NASA Glenn Research Center, Cleveland, OH, USA.

\begin{abstract}
Yttria-stabilized zirconia's high oxygen diffusivity and corresponding high ionic conductivity, and its structural stability over a broad range of temperatures, have made the material of interest for use in a number of applications, for example, as solid electrolytes in fuel cells. At low concentrations, the stabilizing yttria also serves to increase the oxygen diffusivity through the presence of corresponding oxygen vacancies, needed to maintain charge neutrality. At higher yttria concentration, however, diffusivity is impeded by the larger number of relatively high energy migration barriers associated with yttrium cations. In addition, there is evidence that oxygen vacancies preferentially occupy nearest-neighbor sites around either dopant or $\mathrm{Zr}$ cations, further affecting vacancy diffusion. We present the results of ab initio calculations that indicate that it is energetically favorable for oxygen vacancies to occupy nearest-neighbor sites adjacent to $\mathrm{Y}$ ions, and that the presence of vacancies near either species of cation lowers the migration barriers. Kinetic Monte Carlo results from simulations incorporating this effect are presented and compared with results from simulations in which the effect is not present.
\end{abstract}

\section{INTRODUCTION}

Zirconia-based materials, and yttria-stabilized zirconia (YSZ) in particular, are of interest for a variety of technological applications. Pure zirconia exists in a monoclinic structure below about 1100C, with a tetragonal phase stable above that temperature to about 2300C, and a cubic phase stable from there to the melting point [1]. These phase transitions limit the suitability of zirconia for high-temperature applications, particular those that involve frequent thermal cycling.

The tetragonal and cubic phases can be stabilized via substitutional doping, with aliovalent cations such as $\mathrm{Y}^{3+}$ or $\mathrm{Ca}^{2+}$ replacing $\mathrm{Zr}^{4+}$ ions. The cubic phase can be fully stabilized via doping with $\mathrm{Y}^{3+}$, and the resulting yttria-stabilized zirconia remains in the same cubic fluorite phase from room temperature to the melting point. YSZ's thermal stability and low thermal conductivity make it suitable for high-temperature applications such as thermal barrier coatings for turbine engine components.

Oxygen diffusion takes place via diffusive oxygen ion hopping among vacancy sites, with the oxygen ion passing between two barrier cations. When zirconia is cation-doped, additional compensating oxygen vacancies are formed so as to maintain overall electrical neutrality. The addition of these vacancies increases the number of potential hopping sites, increases the diffusivity and the diffusive ionic conductivity, which can attain values large enough to make 
YSZ and related materials of interest for use as oxygen sensors, or as solid electrolytes for fuel cells. However, the oxygen diffusivity does not increase monotonically with $\mathrm{Y}^{3+}$ concentration; it increases at low concentrations, but reaches a maximum between 8 and $15 \mathrm{~mol} \% \mathrm{Y}_{2} \mathrm{O}_{3}$, and decreases at higher concentrations [2].

In addition, there is evidence for oxygen vacancies to exist preferentially as nearest neighbors to either $\mathrm{Zr}$ or Y cations. While this issue has remained unresolved, it is potentially important in that the presence of an oxygen vacancy in the vicinity of one of the barrier cations may affect the value of the migration energy barrier, and thus affect the diffusivity.

In this work we investigate oxygen diffusion in YSZ using a kinetic Monte Carlo (kMC) computer simulation procedure, with barrier energies from density functional calculations. We discuss the temperature dependence of the diffusivity, as well as its dependence on yttria concentration. We also perform density functional calculations of the energetics of the proximity of oxygen vacancies to $\mathrm{Zr}$ and $\mathrm{Y}$ cations, and describe kMC simulations that take into account the modification of migration barrier energies due to the presence of these vacancies.

Fully stabilized YSZ exists in a cubic fluorite structure in which the Zr and Y cations are located at sites on a face-centered cubic sublattice, while the oxygen ions are located on a simple cubic sublattice whose lattice constant is one-half that of the cation sublattice. Oxygen diffusion takes place primarily via the hopping of ions in the [100] direction to nearest-neighbor vacancies on the oxygen sublattice.

In view of the range of potential applications, considerable experimental and theoretical effort has gone into understanding the behavior of YSZ, and, in particular, oxygen diffusion in YSZ and similar materials. Computer simulations using a variety of techniques have been performed. Schelling et al. [3] have investigated the cubic-to-tetragonal phase transition using molecular dynamics simulation, and have correctly predicted the experimentally-observed stabilization due to yttrium doping of $\mathrm{ZrO}_{2}$. Similar work has been carried out by Fabris et al. [4]. Fevre et al. have investigated the thermal conductivity of YSZ using both Monte Carlo [5] and molecular dynamics [6] techniques. Krishnamurthy et al. have performed kinetic Monte Carlo (kMC) simulations of oxygen diffusion in YSZ [7] and similar compounds [8], and have produced diffusivities and diffusivity concentration dependence in reasonable agreement with experiment. Other molecular dynamics studies of oxygen diffusion in YSZ have been performed by by Kahn et al. [9], Okazaki et al. [10], Perumal et al. [11] and Shimojo et al. [12].

\section{THEORY}

Kinetic Monte Carlo simulation [13-15] is a method designed to study systems where so-called "infrequent events" such as diffusive hopping are important. In such systems, molecular dynamics simulation, where the accurate representation of atomic vibrations requires a time step on the order of femtoseconds, are often inefficient, as a large fraction of computational resources are spent simulating the system's behavior in between the events of interest. By contrast, the kinetic Monte Carlo method allows one to concentrate on such events, and to effectively consider only the average behavior of the system between such events, while giving up information on the detailed atomic trajectories. 
In kinetic Monte Carlo simulations of diffusive hopping, the hopping probabilities are determined by the migration barrier energies, that is, the difference in the total energy when the hopping ion is moved from its original position to the saddle point. When the barrier energies are known, the hopping rates $v_{A B}$ may be computed from $v_{A B}=v^{0} \exp \left(-E_{A B} / k_{B} T\right)$ in which $v_{A B}$ and $E_{A B}$ are the hopping rate and migration barrier energy for a hop between oxygen sublattice sites $\mathrm{A}$ and $\mathrm{B}$ respectively, and $v^{0}$ is a frequency factor. $v^{0}$ is typically assigned a value between $10^{12}$ and $10^{13}$ (the value used here) for these materials. For each possible hop, the hopping probability $P_{A B}$ can be computed from the hopping rate, with $P_{A B}=v_{A B} / \Gamma$, where $\Gamma$ is the sum of hopping rates for all possible hops in the computational cell. A catalog of all possible hops, and the corresponding hopping rates and probabilities, is created.

During the kMC process, one of the possible events (that is, a hop defined by the hopping ion, the barrier cation species and the target vacancy site) is chosen probabilistically from the catalog and executed. Hopping probabilities for all possible hops involving the new vacancy location are computed and added to the catalog, while probabilities involving the vacancy's previous location are deleted, and the sum of the hopping probabilities is updated. Finally, the simulation clock is advanced by a stochastically-chosen time step, with $\Delta t=-\ln (R) / \Gamma$, with $0<R \leq 1$ a random number. When the simulation has run for a time $t$ long enough to accumulate statistically useful information, the mean square displacement, averaged over all vacancies, is computed. The vacancy diffusivity $D_{v}$ is obtained from the Einstein relation $\left\langle R^{2}\right\rangle=6 D_{v} t$ and the ionic diffusivity $D_{i}$ is obtained by balancing the number of vacancy and ionic hops, with $D_{i}=C_{V} D_{V} /\left(1-C_{V}\right)$, where $C_{V}$ is the concentration of oxygen vacancies.

The most energetically favorable hopping paths are in the [100] direction, as confirmed by ab initio calculations [7]. The hopping oxygen ion proceeds between two barrier cations to a vacancy site one-half lattice constant away from the original location.

\section{RESULTS AND DISCUSSION}

$\mathrm{Ab}$ initio energy barriers were computed using the Abinit density functional code[16]. There is conflicting evidence that oxygen vacancies preferentially occupy nearest neighbor sites of either $\mathrm{Y}$ or $\mathrm{Zr}$ ions. In order to resolve this ambiguity, we have performed ab initio calculations of the total energy of a 2x2x2 cubic YSZ supercell with an oxygen vacancy in a nearest-neighbor position with respect to $\mathrm{Zr}$ and $\mathrm{Y}$ ions. We find that the $\mathrm{Y}$ nearest neighbor vacancy position is favored over the $\mathrm{Zr}$ nearest neighbor position by $0.20 \mathrm{eV}$. In view of this result, we have.

Table 1. Oxygen migration barrier energies with and without barrier cation nearest neighbor oxygen vacancies.

\begin{tabular}{|l|l|}
\hline Barrier Configuration & Barrier Energy, eV \\
\hline Zr-Zr, no vacancy & 0.706 \\
\hline Zr-Zr, nn vacancy & 0.568 \\
\hline Zr-Y, no vacancy & 1.214 \\
\hline Zr-Y, Zr-nn vacancy & 0.930 \\
\hline Zr-Y, Y-nn vacancy & 0.925 \\
\hline Y-Y, no vacancy & 1.941 \\
\hline Y-Y, Y-nn vacancy & 1.64 \\
\hline
\end{tabular}


computed barrier energies for all possible barrier ion pairs, with and without nearest neighbor vacancies for the barrier cations The results are shown in Table 1. It is evident that there are significant differences in the barrier energies with and without a neighboring oxygen vacancy, ranging from 0.14 to $0.3 \mathrm{eV}$. We have therefore included the vacancy-corrected energies in our simulations stochastically, based on the relative $\mathrm{Zr}$ and $\mathrm{Y}$ concentrations.

The dependence of the oxygen diffusivity on yttria concentration, for a variety of temperatures, is shown in Figure 1. The values of the diffusivity are generally consistent with experiment [18]. The diffusivity is seen to increase at low yttria concentration, reaching a maximum at about $8-25 \% \mathrm{Y}_{2} \mathrm{O}_{3}$, depending on the temperature, and decreasing at higher concentrations. The concentration at which the maximum occurs is seen to increase with increasing temperatures. Both of these points are consistent with observation.

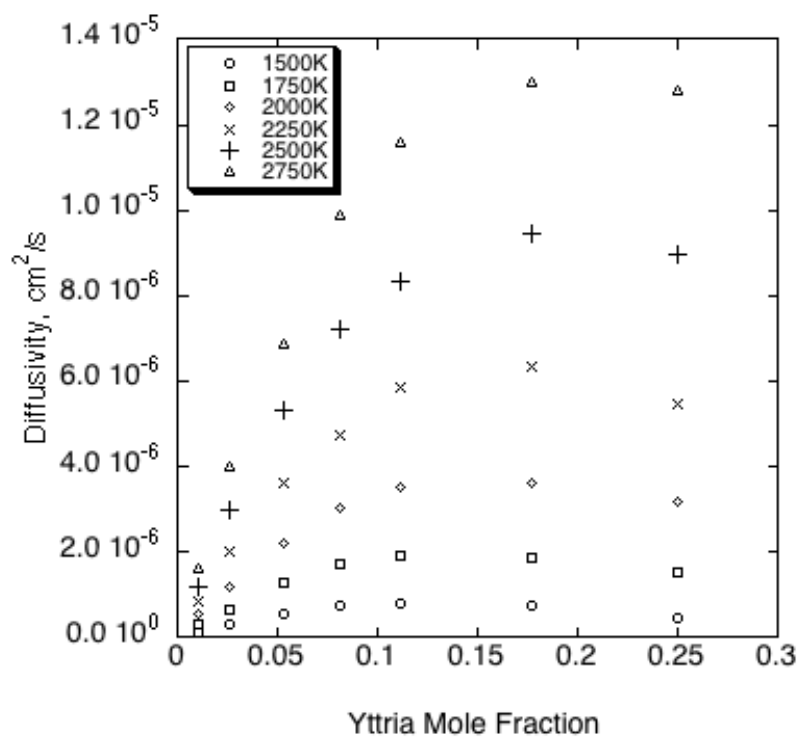

Figure 1. Oxygen diffusivity versus yttria concentration, incorporating barrier cation oxygen vacancy pairing corrections to barrier energies.

The temperature dependence of the diffusivity is shown in Figure 2, along with results from experiment. It can be seen that the values of the kMC diffusivity are reasonably consistent with the experimental results of Oishi [18], though the kMC results exhibit a smaller range of slopes, and hence a smaller range of activation energies. The kMC results generally show an increasing activation energy with yttria concentration, which is consistent with the existence of a larger number of high energy barriers containing one or two $\mathrm{Y}^{3+}$ ions. 


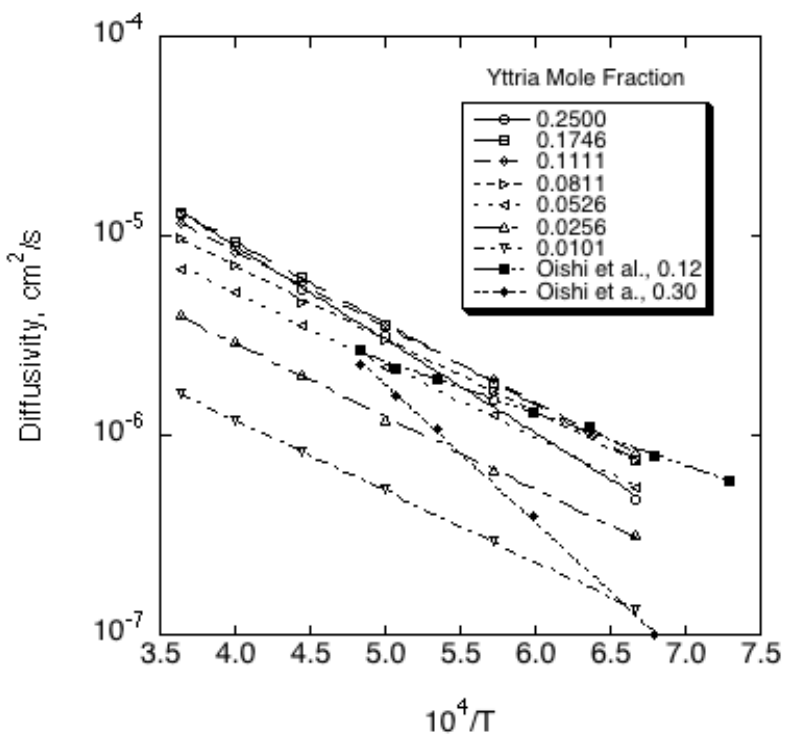

Figure 2. Temperature dependence of diffusivity from kMC results (open icons) and experiment (solid icons).

The concentration dependence of the diffusivity, with and without barrier cation vacancy pairing, is shown in Figure 3. It can be seen that in both cases the diffusivity at a given temperature is larger for the cases incorporating oxygen vacancies, consistent with the lower barrier energies in such cases. The differences are very small for $\mathrm{T}=1500 \mathrm{~K}$, but the maximum difference in the $\mathrm{Y}=2500 \mathrm{~K}$ diffusivities is approximately fifteen percent. The location of the diffusivity maximum with concentration does not appear to change significantly when vacancy pairing is included. It is also found that the variation in the temperature dependence with and without vacancy pairing is very small.

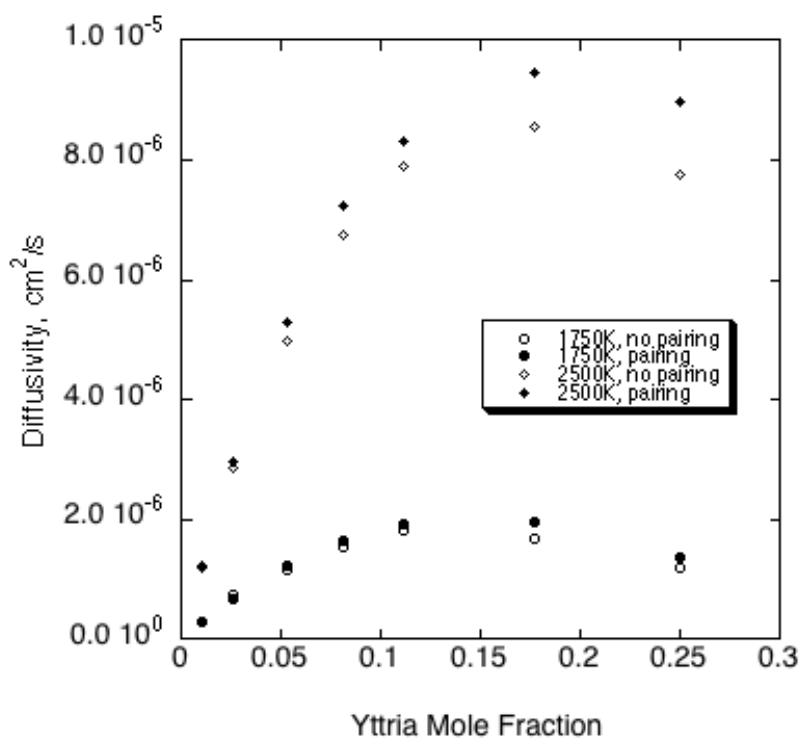

Figure 3. Oxygen diffusivity versus yttria concentration, with (solid icons) and without (open icons) barrier cation oxygen vacancy pairing. 


\section{CONCLUSIONS}

We have performed kinetic Monte Carlo computer simulations of oxygen diffusion in yttria stabilized zirconia. Isolated oxygen vacancies in a nearest-neighbor configuration with respect to a $\mathrm{Y}^{3+}$ cation are energetically favored by $0.2 \mathrm{eV}$ compared with a nearest-neighbor position with

respect to a $\mathrm{Zr}^{4+}$ cation. Incorporating oxygen vacancies as nearest neighbors of either of the cations in a barrier pair results in a lowering of the barrier energies by 0.14 to $0.3 \mathrm{eV}$, with a resulting increase in oxygen diffusivity, though the effect is only significant at high temperatures and high yttria concentrations. Oxygen diffusivities computed using barrier energies from density functional calculations, with and without vacancy pairing, are consistent in magnitude with results from experiment and from other simulations. The dependences of the diffusivity on $\mathrm{Y}^{3+}$ concentration and on temperature are also consistent with experiment.

\section{REFERENCES}

1. P. Aldebert and J. P. Traverse, J. Am Ceram. Soc. 68 [1], 34-40 (1985).

2. R. E. W. Casselton, Phys. Status Solidi A, 2, 571-585 (1970).

3. P. K. Schelling, S. R. Phillpot and D Wolf, J. Am Ceram. Soc. 84 [7], 1609-1619 (2001).

4. S. Fabris, A. T. Paxton and M. W. Finnis, Phys. Rev. B 63, 094101 (2001).

5. M Fevre, A. Finel and R. Caudron, Phys. Rev. B 72, 104117 (2005).

6. M Fevre, A. Finel, R. Caudron and R. Mevrel, Phys. Rev. B 72, 104118 (2005).

7. R. Krishnamurthy, Y.-G. Yoon, D. J. Srolovitz and R. Car, J. Am. Ceram. Soc. 87 [10],18211830 (2004).

8. R. Krishnamurthy, D. J. Srolovitz, K. N. Kudin and R. Car, J. Am. Ceram. Soc. 88 [8],21432151 (2005).

9. M. S. Kahn, M. S. Islam and D. R. Bates, J. Mater. Chem. 8 [10], 2299-2307 1998.

10. H. Okazaki, H. Suzuki and K. Ihata, Phys. Let. A 188, 291-295 (1994).

11. T. P. Perumal, V. Sridhar, K. P. N. Murthy, K. S. Easwarakumar and S. Ramasamy, Comp. Mat. Sci. 38, 865-872 (2007).

12. F. Shimojo, T. Okabe, F. Tachibana, M. Kobayashi and H. Okazaki, J. Phys. Soc. Jpn 61, 2848-2857 (1992), and F. Shimojo and H. Okazaki, J. Phys. Soc. Jpn 61, 4106-4118 (1992).

13. W. M. Young and E. W. Elcock, Proc. of the Phys. Soc. 89, 735 (1966) .

14. A. B. Bortz and M. H. Kalos and J. L. Lebowitz, J. of Comput. Physics 17, 10 (1975).

15. A. F. Voter, Kinetic Monte Carlo, in Radiation Effects in Solids, Proceedings of the NATO Advanced Study Institute on Radiation Effects in Solids, K. E. Sickafus, E. A. Kotomin, B. P. Uberuaga, eds., 1-23, Springer, Dordrecht, The Netherlands, 2007.

16. X. Gonze, B. Amadon, P.-M. Anglade, J.-M. Beuken, F. Bottin, P. Boulanger, F. Bruneval, D. Caliste, R. Caracas, M. Cote, T. Deutsch, L. Genovese, Ph. Ghosez, M. Giantomassi, S. Goedecker, D. R. Hamann, P. Hermet, F. Jollet, G. Jomard, S. Leroux, M. Mancini, S. Mazevet, M. J. T. Oliveira, G. Onida, Y. Pouillon, T. Rangel, G.-M. Rignanese, D. Sangalli, R. Shaltaf, M. Torrent, M. J. Verstraete, G. Zerah, J. W. Zwanziger, Computer Phys. Comm. 108, 2582 (2009).

17. X. Li and B. Hafskjold, J. Phys.: Condens. Matter 7, 1255 (1995).

18. Y. Oishi and K. Ando, Transport in Nonstoichiometric Compounds, G. Simovich and V. Stubican, eds., NATO ASI Ser. B 129, 1985.

19. I. R. Gibson and J. T. S. Irvine, J. Mater. Chem. 6, 895 (1996).

20. A. Orliukas, P. Bohac, K. Sasaki and L. J. Gauckler, Solid State Ionics 72, 35 (1994).

21. I. R. Gibson, E. E. Lachowski, J. T. S. Irvine and G. P. Dransfield, Solid State Ionics 72, 265 (1994). 